\title{
INVESTIGACION
}

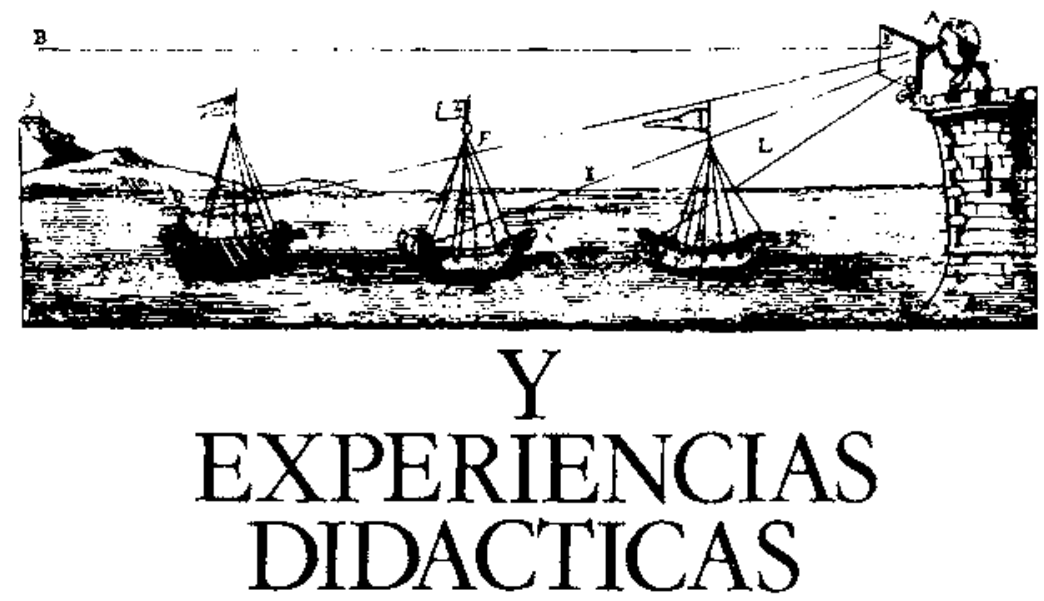

\section{CONCEPCIONES DE LOS ESTUDIANTES SOBRE UNA MAGNITUD «OLVIDADA» EN LA ENSEÑANZA DE LA QUÍMICA: LA CANTIDAD DE SUSTANCIA}

FURIÓ, C. ${ }^{1}$, AZCONA, R.. ${ }^{2}$, GUISASOLA, G. ${ }^{3}$ y MUJIKA, E. ${ }^{4}$

${ }^{1}$ Departament de Didàctica de les Ciències Experimentals. Universitat de València.

${ }^{2}$ IB de Hondarribia (Guipúzcoa).

${ }^{3}$ Departamento de Física Aplicada I. Universidad del País Vaseo.

4 Lizeo Pasaia (Guipúzcoa).

\section{SUMMARY}

The main objective of this paper is the identification of the students' representations about the idea of substance quantity and to see to what extent the establised Chemistry teaching in Secondary schools contributes to giving them a more atomist view of matter. The results obtained in this work confirm that students, upon completing their studies, have practically not changed their 'globalist' conception of substance quantity, and only half of those that studied chemistry master the necessary activity to be able to understand the mole concept.

\section{INTRODUCCIÓN}

El aprendizaje significativo del concepto de mol en los cursos introductorios de química es un problema sin resolver a pesar de la abundante bibliografía sobre esta temática (Dierks 1980) y que con frecuencia aparece en Ia literatura didáctica (Grupo Alkali 1990, Ainley 1991, Nelson 1991). Se detectan en el aprendizaje de este 
concepto gran cantidad de errores conceptuales (Caamaño et al. 1983) y las razones a las que se achacan las dificultades de su enseñanza son muy diversas, como:

a) las dificultades intrínsecas de comprensión que representa para los estudiantes de secundaria la definición rígurosa de la IUPAC, bien por la elevada exigencia cognitiva del propio concepto (Shayer y Adey 1984), bien por los prerrequisitos matemáticos de pensamiento proporcionai necesarios para su asimilación (Rowell y Dawson 1980)...;

b) la presentación inadecuada de este concepto en los Iibros de texto (de Berg 1986b) o Ias deficientes metodologías utilizadas por el profesorado en la enseñanza habitual (de Berg 1986a).

Desde un punto de vista didáctico hay que tener en cuenta que el mol es la unidad de la magnitud cantidad ce sustancia y parece evidente que la introducción de este último concepto ha de ser previa al de mol. Sin embargo, Ias estrategias de enseñanza habituales, basadas en un operativismo extremo cuando se introducen conceptos químicos, apenas inciden en la cantidad de sustancia y pasan directamente al concepto de mol. Es,

Figura 1

El referente empirico $\left(R_{1}\right)$ y el referente atomista $\left(R_{2}\right)$ en los que se introduce el concepto de cantidad de sustancia.

REPEREATE GCPÍRTCO $\left(\mathbf{R}_{\mathfrak{l}}\right)$

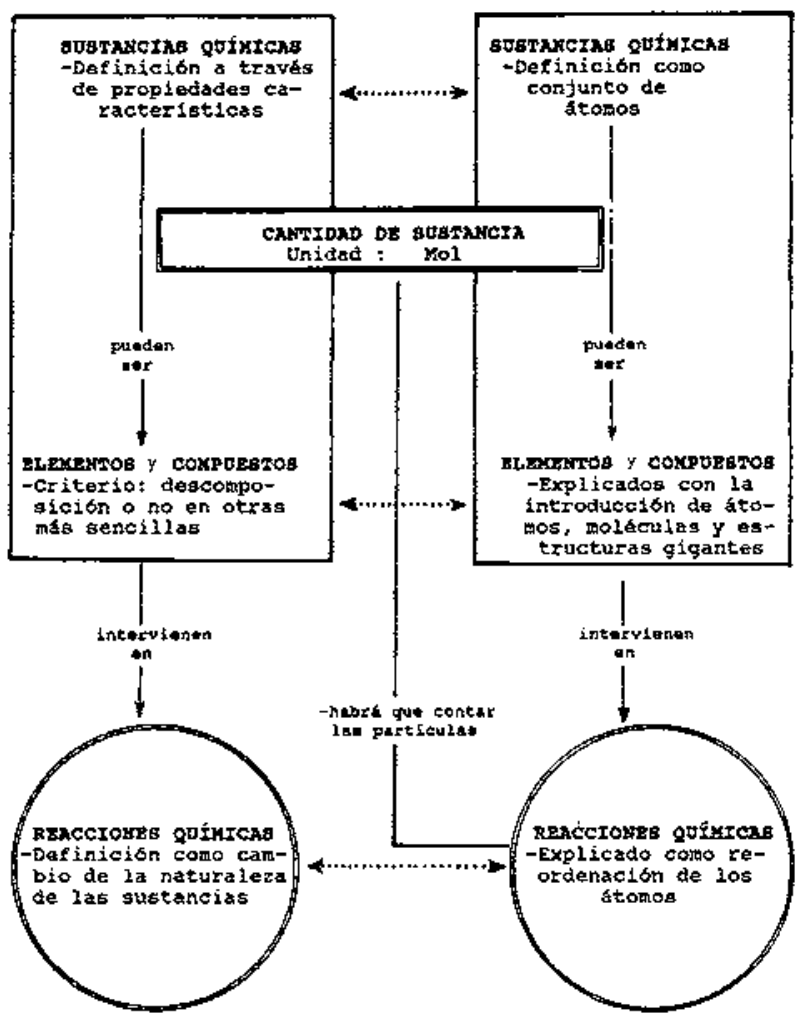

pues, evidente que, si se quiere enseñar el mol desde una perspectiva constructivișta del aprendizaje, será necesario averiguar previamente cuáles son las ideas que tienen los estudiantes sobre el concepto cualitativo de cantidad de sustancia. Ideas que, a su vez, dependerán en mayor o menor medida de la visión que aquéllos posean sobre Ia naturaleza continua o discontinua de la materia.

Así pues, el objetivo esencial de este trabajo será diagnosticar cuáles son las representaciones mentales que tienen los estudiantes sobre la idea de cantidad de sustancia y ver en qué medida la formación química recibida en la enseñanza secundaria contribuye a darles una visión más significativa de la misma. A nuestro entender, es muy probable que estas ideas, previas de los estudiantes puedan actuar como obstáculo epistemológico si no se contempla la necesidad lógica de su introducción y, sobre todo, si no se diferencia progresivamente de otras propiedades generales de la materia como son Ia masa y el volumen (Otero 1985). Solamente teniendo en cuenta aquellas ideas, se podrán abordar estrategias didácticas eficaces que puedan resolver el problema que supone un aprendizaje significativo de dicho concepto sin el cual no se puede comprender el de su unidad, el mol.

\section{EL PROBLEMA DE FONDO: ¿SON ATOMISTAS O GLOBALISTAS LOS ESTUDIANTES DE QUIMICA QUE ACABAN LA SECUNDARIA?}

La necesidad de introducir los conceptos de cantidad de sustancia y de su unidad, el mol, dentro del contexto químico de la teoría atómica de Ia materia se deriva de las explicaciones que allí se dan a los conceptos de sustancia química -formadas por partículas- y al de reacción química como redistribución de átomos (Fig. 1). Pero, cabe destacar que, en general, al introducir estos conceptos, el profesor no diferencia suficientemente (más bien los mezcla sin saberlo) los dos sistemas de referencia epistemológica reseñados por la psicología cognitiva: 1) el referente empírico, cuya visión macroscópica podemos entender como un globalismo $(R$, en la figura 1), ya que fija su atención en aquellas propiedades de las sustancias (más bien de la materia) vistas como un todo común (sin partes) y que se concretan en conceptos como la masa, el peso o el volumen bastante interiorizadas (aunque, muchas veces, no diferenciadas) en los estudiantes de secundaria a través de procesos de generalización inductiva; y 2) el referente atomista $\left(\mathrm{R}_{2}\right)$ como visión submicroscópica que explica estos procesos químicos con conceptos como la cantidad de átomos mediante procesos de abstracción reflexiva o de generalización constructiva (Vuik 1981). La introducción de la idea de cantidad de sustancia se derivaría de la necesidad de establecer las oportunas relaciones entre los dos referentes. En efecto, la interpretación cuantitativa de cualquier proceso químico implica la necesidad de contar las partículas intervinientes de las distintas sustancias independientemente de la masa es- 
pecífica de sus partículas. El problema quedaría resuelto, desde el punto de vista epistemológico, con la introducción de la cantidad de partículas. No obstante, la imposibilidad material de contarlas directamente y la necesidad -iy comodidad!- de hacerlo a través del otro referente empírico obliga a introducir esta nueva magnitud, la cantidad de sustancia, que servirá como magnitud macroscópica para comparar cantidades de partículas en porciones de sustancias diferentes, y que no debe confundirse ni con la masa muy conocida por los estudiantes que se inician en la Química, ni con la cantidad de partículas, confusión que se da frecuentemente en el profesorado como veremos más adelante. Así pues, el atributo principal de la cantidad de sustancia en este contexto teórico será el de porción de sustancia que contiene un número de conjuntos o «paquetes» de partículas y, en una primera aproximación cualitativa, podemos establecer que la asociación de ideas entre cantidad de sustancia y cantidad de partículas será la propia de una visión atomista de la materia (más adelante se podrán diferenciar progresivamente las magnitudes cantidad de partículas y cantidad de sustancia). Por el contrario, en el caso de que se perciban prioritariamente las sustancias como un todo y, en consecuencia, se asocie preferentemente la idea de cantidad de sustancia con la de masa -más bien, con la de peso- o con la de volumen, entenderemos que predomina un perfil globalista primario sobre la constitución de la materia (Bachelard 1978).

En este contexto, el primer problema que nos planteamos en este trabajo fue averiguar por cuál de los tipos de asociaciones de ideas, la atomista o la/s globalista/s, antes descritas, se inclinan las preferencias de nuestros estudiantes al iniciarse en el estudio de la química y cuál es su evolución a lo largo del bachillerato y COU con el fin de comprobar la influencia de aquellos estudios en una adquisición de un punto de vista más atomista de la materia.

Teniendo en cuenta la poca eficacia, en general, de la enseñanza de los conceptos científicos debida, en particular, a la escasa atención que el profesorado presta a las ideas previas del alumnado y, en este caso, la nula que se destina a la introducción del concepto de cantidad de sustancia (ni siquiera aparece como contenido en los textos), de todo ello se infiere como hipótesis que la mayoría de los estudiantes tendrá inicialmente un punto de vista predominantemente globalista de la materia y que la evolución del mismo, a lo largo de los estudios de química en el bachillerato, apenas sufrirá variación, no observándose ningún cambio conceptual significativo hacia una iđea cualitativa de cantidad de sustancia más acorde con una visión atomista de la materia.

Por otra parte, una introducción cualitativa y significativa del concepto de cantidad de sustancia requiere, como primer paso, identificar la proporción en masa de distintas sustancias que tengan cantidades iguales de partículas teniendo presente que las respectivas masas que las caracterizan son diferentes. Esto significa, desde un punto de vista atomista, mantener invariante en aquellas porciones el número de partículas $y$, en consecuencia, comprender que hay que tomar masas de las diferentes sustancias en la misma proporción directa que las de las masas atómicas, moleculares o fórmulas respectivas. En cambio, desde un punto de vista globalista, cantidades iguales de sustancia significarán mantener mentalmente constante la masa de las sustancias y, lógicamente, se necesitarán más partículas que tengan menor masa específica para tener una masa total dada (proporcionalidad inversa entre la masa de las diferentes sustancias que se toman y la masa característica de sus respectivas partículas). Razonamientos análogos se pueđen hacer considerando la relación entre los volúmenes totales de sustancias y los volúmenes característicos de cada entidad elemental (sea átomo o molécula).

Éste ha sido el segundo problema abordado en este trabajo: conocer en qué medida los estudiantes antes y después de haber estudiado química en la enseñanza secundaria dominaban esta actividad mental en casos concretos donde las masas o los volúmenes de las partículas que formaban las sustancias estuvieran en una razón matemática sencilla (para que no hubiera dificultades en este dominio). En realidad, esta tarea de aprendizaje se caracteriza por poseer cierta complejidad cognitiva, ya que su solución correcta no sólo implica cierta destreza de pensamiento proporcional sino que además supone, y esto a nuestro entender es lo más importante, cierto dominio del contex to atomista, pues se debe estabiecer una relación entre los dos sistemas de referencia indicados y, más en concreto, entre la masa de la sustancia como un todo (referente empírico-globalista) y la masa de la misma concebida como la suma de las masas de todas las partículas que la forman (referente atomista). Nuestra hipótesis respecto a este problema es que existirá una influencia curricular positiva en los alumnos de BUP y de COU que hayan estudiado química respecto de los que no lo han hecho, debido a que están más familiarizados con el contexto químico daltoniano y, en particular, a que han manejado muchas más veces los conceptos de átomo y de masa atómica, si bien de forma memorística y operativista. De la aceptación de esta hipótesis, se deduce que, si en esta actividad mental mantenemos invariable la complejidad matemática (la razón de proporcionalidad empleada) y aumentamos la familiaridad del contexto (p.e. sustituyendo los conjuntos de átomos de distintos elementos por conjuntos de frutas distintas en carda uno de los cuales hay entidades elementales idénticas en masa), debe lograrse mayor éxito en las respuestas, y no sólo en estudiantes de química sino incluso en aquellos otros de las mismas edades y centros pero de Letras.

\section{DISEÑOS EXPERIMENTALES}

Para contrastar las hipótesis anteriores se ha diseñado un cuestionario con varias partes en forma de preguntas de opción múltiple con dibujos. En la primera parte, que contiene tres ítems (véase prueba 1 en el anexo I), se trata de averiguar no sólo cuál es la preferencia cognitiva de los estudiantes respecto a la idea de cantidad de sustancia, sino también si cada uno de aquéllos responden de forma coherente con el mismo esquema conceptual a los tres ítems. Para ver por cuál de las tres posibles asociaciones 
de ideas se decantan, se les presenta en cada ítem dos sustancias, con sus átomos simulados, sobre los platillos de una balanza y se les pregunta dónde hay mayor cantidad de sustancia, solicitando una explicación de sus respuestas. En cada uno de los ítems se ha mantenido constante una de las tres variables (número de partículas, peso y volumen), respectivamente. Las respuestas se han clasificado de acuerdo con el tipo de asociación de ideas elegido, es decir, en:

a) esquemas conceptuales que asocian cantidad de sustancia con peso,

b) esquemas conceptuales que asocian cantidad de sustancia con volumen,

c) esquemas conceptuales que asocian cantidad de sustancia con cantidad de partículas.

La segunda parte del cuestionario contiene dos ítems. En el primero de ellos se trata de averiguar los pesos que deberían cogerse de dos elementos sólidos diferentes (cobre y azufre) para poder tener el mismo número de átomos, conociéndose los pesos relativos de aquellos átomos (véase prueba 2 del anexo I). Aquí figurarán, como distractores, una respuesta atomista correcta, para una razón de proporcionalidad sencilla (2/1), y otra coherente con un pensamiento empírico-globalista, para la razón inversa (1/2). Por lo que respecta al segundo ítem de esta parte, es muy semejante al anterior pero se ha cambiado el peso por el volumen, los elementos por compuestos, el estado sólido de las sustancias por el líquido y el tipo de partículas (moléculas por átomos).

Estas pruebas se han aplicado a estudiantes de los tres cursos de bachillerato, de COU de Química y de COU de Letras en varios centros de bachillerato con el fin de observar la evolución de las concepciones atomista y globalistas a través de sus estudios secundarios.

Finalmente, para estudiar la influencia de la familiaridad del contexto en la segunda parte, se ha ideado otro ítem en el que se ha eliminado toda mención química y se ha contextualizado la pregunta en un ambiente cotidiano, donde en lugar de átomos distintos se toman naranjas y manzanas. Esta pregunta se aplicaría a otros dos nuevos grupos de COU de Ciencias y de Letras, respectivamente. En conjunto la muestra a los que se ha aplicado todo o parte del cuestionario ha sido de 610 alumnos de institutos y centros privados de secundaria de Guipúzcoa.

\section{RESULTADOS Y ANÁLISIS DE LOS MISMOS}

En la tabla I se presentan los porcentajes de la coherencia en las respuestas de todos los alumnos, así como los de las coherencias en cada uno de los tipos de esquemas conceptuales globalistas ( $a$ y $b$ ) y atomista $(c)$ considerados en función del nivel educativo. También figuran entre paréntesis estos porcentajes pero tomando como base de cálculo el total de respuestas coherentes. De estos resultados se ha representado, por una parte, la
Figura 2, donde se puede analizar más sencillamente la evolución de la concepción atomista de la cantidad de sustancia a lo largo del bachillerato. En efecto, puede comprobarse que, de todos los alumnos encuestados, solamente alrededor de un $15 \%$ tiene este esquema conceptual y, lo que es más grave, este porcentaje se mantiene prácticamente constante $e$ independiente del número de cursos de química recibidos en sus estudios de secundaria. En base a ello podemos afirmar que estas enseñanzas en el bachillerato apenas contribuyen a elevar el porcentaje de alumnos que asocian cualitativamente la cantidad de sustancia con el número de partículas.

Tabla 1

Porcentajes de coherencia global y de coherencia en cada uno de los esquemas conceptuales de cantidad de sustancia en funcion del nivel educativo de los estudiantes

\begin{tabular}{lccccc}
\hline Grupo & N & Coherencia & \multicolumn{4}{c}{ Esquemas conceptuales coh.(\%) } \\
& & global(\%) & a & $b$ & $c$ \\
\hline I BUP $^{2}$ & 90 & $61(100)$ & $38(62,3)$ & $12(19,7)$ & $11(18,0)$ \\
$2^{\circ}$ BUP & 116 & $69(100)$ & $37(53,6)$ & $13(18,8)$ & $19(27,5)$ \\
$3^{\circ}$ BUP Q & 191 & $70(100)$ & $28(40,0)$ & $31(44,3)$ & $11(15,7)$ \\
COU Q1 & 142 & $70(100)$ & $17(24,3)$ & $34(48,6)$ & $19(27,1)$ \\
TOTAL & 539 & $69(100)$ & $29(42,0)$ & $25(36,2)$ & $15(21,7)$ \\
COU L1 & 43 & $47(100)$ & $16(34,0)$ & $19(40,4)$ & $12(25,5)$ \\
\hline
\end{tabular}

Figura 2

Evolución de la concepción atomista de cantidad de sustancia a lo largo del bachillerato.

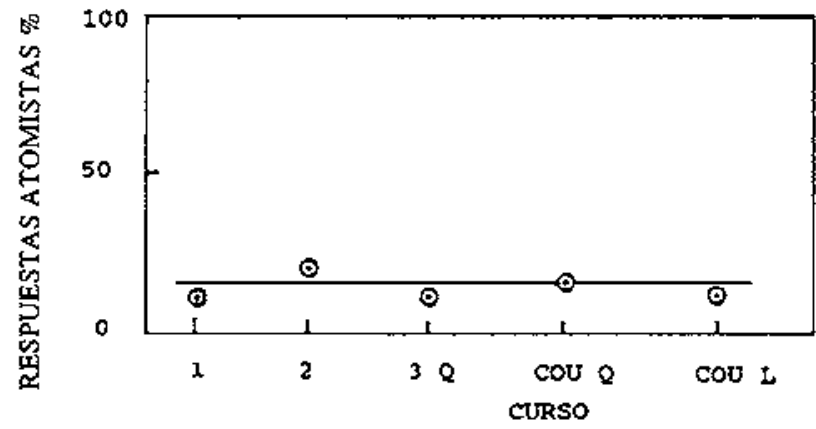

Al mismo tiempo, para facilitar el análisis de Ia evolución de los esquemas conceptuales globalistas de la cantidad de sustancia, se han representado en Ia figura 3 los porcentajes de los esquemas $a, b$ y $a \circ b$ en función del curso. Podemos observar que coherentemente con el resultado anterior se mantienen constantes y próximos al $80 \%$ de respuestas coherentes la suma de porcentajes de esquemas globalistas. Ahora bien, existe una disminución progresiva, desde $1^{\circ}$ de BUP hasta $3^{\circ}$ y COU, del 
porcentaje de alumnos que asocian la «cantidad de sustancia» con el peso y un aumento simultáneo de los que to hacen con el volumen. Una posible explicación a este hecho podemos encontrarla en que el peso (o la masa) es la primera característica sustancial que se atribuye a la materia corpórea en el pensamiento ordinario, dejando en segundo lugar el tamaño o volumen de los objetos (Bachelard 1978). Por otra parte, estos resultados son congruentes globalmente con los datos empíricos conocidos de estudios piagetianos sobre Ia evolución psicológica del pensamiento de los niños, en los que se ha evidenciado que la adquisición de la conservación del volumen en procesos de transformación de forma en sólidos y líquidos es posterior a la de la conservación del peso en aquellos mismos procesos (Piaget 1970).

Figura 3

Evolución de las concepciones globalistas de cantidad de sustancia a lo largo del bachillerato.

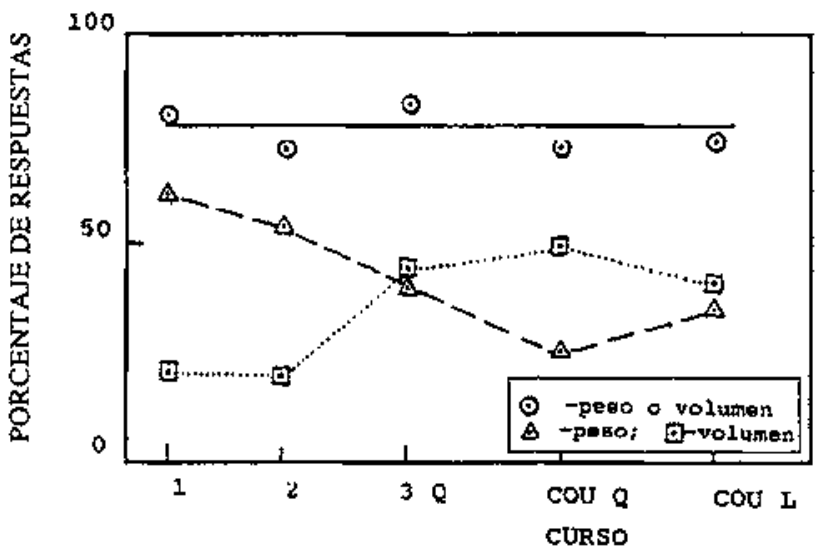

En relación con la segunda parte de este estudio se detallan en la tabla II los resultados encontrados en forma de porcentajes de respuesta a cada uno de los distractores propuestos en las pruebas 2 y 3 mencionadas en el apartado anterior. En la Figura 4 se han representado, en ambas pruebas, sólo los porcentajes de acierto -según el punto de vista atomista- en la relación de pesos (o de volúmenes) que había que tomar de las dos sustancias para tener la misma cantidad de átomos (o de moléculas) en función del curso.

Un análisis detenido de esta gráfica permite contrastar que, en general, existen prácticamente dos poblaciones escolares diferentes: una con los alumnos de $1^{\underline{9}}, 2^{\circ}$ de BUP y COU de Letras que aciertan en un promedio aproximado y constante del $23 \%$ de la muestra a estas actividades, y otra con los estudiantes de $3^{\circ}$ y COU de Química con un $52 \%$ aproximado de contestaciones correctas. Es decir, se comprueba que existe una influencia curricular positiva, debida, sin duda, a la mayor experiencia de estos alumnos en el contexto químico y ello hace que se duplique el porcentaje de éxito. No obstante, queda aún la mitad de estos alumnos que en un nivel de Química de COU no acierta en esta actividad. Como puede verse no hay prácticamente diferencias en
Tabla II

Porcentajes de respuesta a los distractores de las pruebas 2 y 3 de! cuestionario en función del curso.

\begin{tabular}{|c|c|c|c|c|c|c|c|c|c|}
\hline Curso & $N$ & & Pruet & ba $2(9$ & & & Prueb & $3(\%)$ & \\
\hline & & $=$ & $2 \mathrm{Cu}^{*}$ & $2 S$ & $?$ & $=$ & $2 \mathrm{H}_{2} \mathrm{O}$ & $2 X(*)$ & \\
\hline $1^{8} \mathrm{BUP}$ & 90 & 4,4 & 21,1 & 60.0 & 14,4 & 4,5 & 51,7 & 27,0 & 16,8 \\
\hline $2^{\circ} \mathrm{BUP}$ & 116 & 8.6 & 18,1 & 60,3 & 12,9 & 6,0 & 50,9 & 25,9 & 17,2 \\
\hline $3^{\circ} \mathrm{BUP} Q$ & 191 & 5,2 & 48,2 & 40.8 & 5,7 & 3,7 & 38,4 & 48,9 & 8,9 \\
\hline COU Q1 & 142 & 4.2 & 51,4 & 42,2 & 2,1 & 6,3 & 31,7 & 57,0 & 4,9 \\
\hline COUL1 & 43 & 7,1 & 23,8 & 47,6 & 21,4 & 9,3 & 48,8 & 23,2 & 18,6 \\
\hline
\end{tabular}

* Respuesta correcta

$=:$ igual peso

? : no lo sé

Figura 4

Porcentaje de aciertos en la relación de pesos (o volúmenes) que hay que tomar para tener la misma cantidad de sustancia en función del curso.

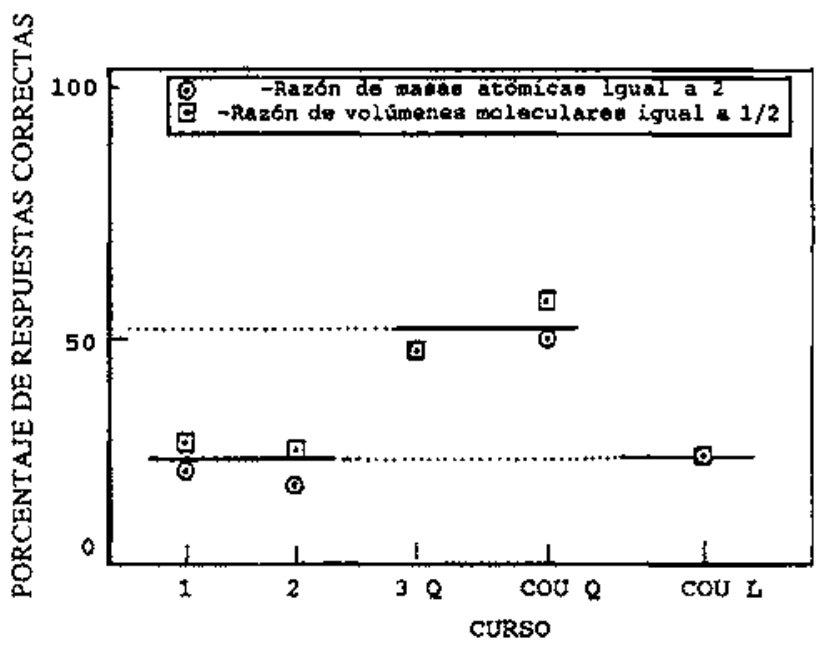

las respuestas cuando en las pruebas se han empleado volúmenes en lugar de pesos, ni tampoco cuando se han sustituido los átomos por moléculas en cada uno de los cursos que se han encuestado. Al propio tiempo, debe destacarse que los estudiantes de COU de Letras obtienen resultados casi idénticos a los de $1^{g}$ y $2^{g}$ de BUP, lo que es síntoma de validez interna de estos resultados, ya que todos estos estudiantes tienen en común que están en el mismo nivel en cuanto a estudios de química recibidos.

Finalmente, en la tabla III, se han expresado los porcentajes de éxito de nuevos estudiantes de COU de Ciencias (COU Q2) y de Letras (COU L2) encuestados en un contex to ordinario mucho más conocido que el químico al que se refería la prueba 2 . En la figura 5 se han 
representado los porcentajes de éxito de estos alumnos y los obtenidos previamente por los anteriores grupos de COU (COU Q1 y COU L1) para poder comparar la influencia de la familiaridad del contexto de la prueba con el éxito de las respuestas a la misma, manteniendo las mismas exigencias matemáticas, tanto en los alumnos de Ciencias como en los de Letras.

Tabla III

Porcentaje de respuestas de alumnos de COU de Ciencias y de Letras a una prueba similar a la 2 dentro de un contexto ordinario.

\begin{tabular}{lccccc}
\hline Curso & N & $\begin{array}{c}\text { Pgual peso } \\
\text { de ambas }\end{array}$ & $\begin{array}{c}\text { Doble peso } \\
\text { naranjas* }\end{array}$ & $\begin{array}{c}\text { Doble peso } \\
\text { manzanas }\end{array}$ & No lo sé \\
\hline COU L2 & 28 & 0 & 46,4 & 39,3 & 14,3 \\
COU Q2 & 26 & 0 & 65,0 & 31,0 & 4,0 \\
\hline
\end{tabular}

* Respuesta correcta

Como puede apreciarse, los alumnos de Letras han pasado de un $23 \%$ a un $46 \%$ de éxito y, al mismo tiempo, en los de Ciencias el cambio ha sido bastante significativo, pues se ha elevado el promedio del porcentaje de aciertos del $52 \%$ al $65 \%$. En definitiva, ha quedado comprobado que la familiarización de los estudiantes en el contexto de la tarea que se propone es una variable digna de tener en cuenta a la hora de planificar actividades en la enseñanza-aprendizaje de la química y es evidente, a la vista de estos resultados, la influencia positiva que puede ejercer el uso de analogías, como la empleada en la última pregunta, con la finalidad práctica de aproximar al estudiante hacia estos conceptos abstractos que manejamos en ciencias.

Figura 5

Porcentaje de aciertos de alumnos de COU de Ciencias y de Letras en ja relación de pesos que hay que tomar para tener la misma

cantidad de unidades en un contexto ordinario y en otro químico.

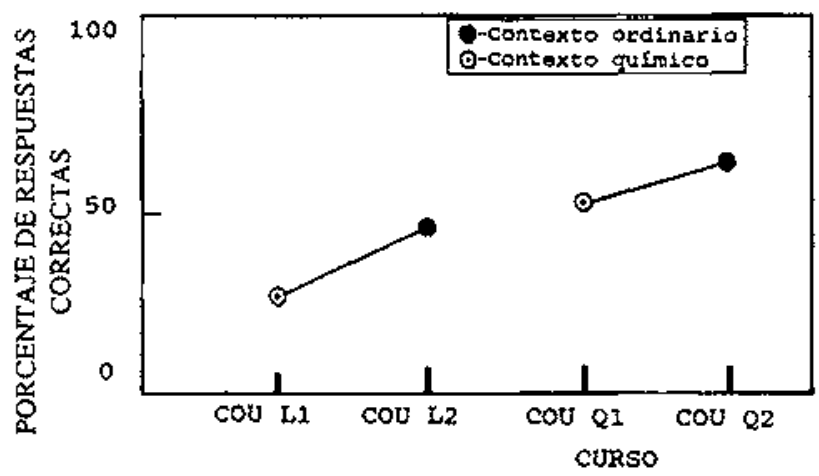

\section{CONCLUSIONES E IMPLICACIONES DIDÁCTICAS}

En resumen, los resultados obtenidos en este trabajo confirman que los estudiantes al acabar sus estudios de secundaria no han cambiado prácticamente su concepción cualitativa globalista de la magnitud cantidad de sustancia y sólo la mitad de los que han estudiado química dominan una actividad básica para poder comprender el concepto de mol. Al mismo tiempo se ha constatado la importancia que tiene familiarizar al estudiante en el contex to de la tarea para poder tener exito en la misma. A partir de estos resultados se abren nuevos interrogantes e implicaciones didácticas que pueden ayudar a cambiar las formas habituales en que se enseña el concepto de mol.

En efecto, que los estudiantes de secundaria avanzada (17-18 años), ya iniciados en química, no diferencien entre cantidad de sustancia y las nociones de masa y volumen es una consecuencia lógica que se deriva de la ausencia general de aquel contenido en la enseñanza de la química. Pero, no lo es que casi todo el profesorado enseñe el concepto de mol, incluso en edades demasiado tempranas (Shayer y Adey 1981), introduciendo la definición rigurosa dada por la IUPAC (Mills et al. 1988) como unidad de cantidad de sustancia y, sin embargo, no se preste la mínima atención a esta magnitud química.

Surge, pues, una primera cuestión didáctica: ¿por qué no se enseña la cantidad de sustancia? En nuestra opinión, la hipótesis más plausible, que trataremos de fundamentar, va en el sentido de que el profesorado no ve la necesidad lógica de introducir este concepto a través de alguno de estos dos argumentos, que en defintiva constituyen confusiones epistemológicas: $a$ ) se supone que la cantidad de sustancia es sinónima de cantidad de materia expresada en términos de masa; $b$ ) se identifica la cantidad de sustancia con la de entidades elementales.

Un síntoma general del primer error citado puede apreciarse cuando se analizan las respuestas de ejercicios estequiométricos presentados en la enseñanza de la química al plantear cuestiones como ¿qué cantidad de cloruro sódico se obtendrá...? La gran mayoría de estas respuestas están contextualizadas en el referente empí rico (físico) y se responden con el cálculo final de la masa, cuando en realidad se deberían contextualizar coherentemente en el referente atomista (químico) y finalizar el cálculo con la cantidad de sustancia expresa. da en moles. Por otra parte, la ambigitedad terminológica que existe en el pensamiento ordinario entre las expresiones cantidad de materia y cantidad de sustancia (Llorens 1991) refuerza su identidad semántica y facilita la confusión. En este sentido, conviene apoyar un carm. bio de nombre para esta última magnitud pero, opina. mos, que el término «cantidad química» recientemente propuesto (Neison 1991) no parece muy acertado desde el punto de vista semántico. Mas adecuada nos parecería «cantidad de sustancia química» que, por una parte, sesgaría su significado hacia el referente atomista y, por otra, diferenciaría más las palabras materia y sustancia en el referente empírico. 
Una segunda confusión que también se da frecuentemente en la enseñanza de la química es la identificación entre cantidad de sustancia (n) y la cantidad de partículas $(\mathrm{N})$ en el sentido de que se considera al primer concepto como derivado (una especie de múltiplo) del segundo. Es decir, desde el punto de vista epistemológico, no se ve la necesidad lógica de introducir una nueva magnitud química cuando ya se ha construido como tal el número de partículas. Así es como se percibe la cantidad de sustancia cuando se utiliza la metáfora de que el mol es la «docena» del químico. Se olvida que la introducción de conceptos a título de hipótesis algunas veces, comoen este caso, no obedece a reglas de tipo lógico-racional (Toulmin 1977), sino más bien a la comodidad que supone para el químico considerar macroscópicamente porciones de sustancia que tengan Ia misma cantidad de partículas. Otro ejemplo similar más conocido es la introducción del concepto de $\mathrm{pH}$ cuya construcción se debe a la comodidad de su manejo operativo frente al de las pequeñas concentraciones de iones hidroxonio existentes en las soluciones acuosas. Más aún, a este respecto conviene recordar que la introducción a principios de siglo de la palabra «mol» por el químico-físico Ostwald se debe a que la prefirió a la entonces existente de «molécula-gramo» porque, según él, no había suficientes evidencias cientufficas sobre la existencia de las moléculas. El significado que le atribuyó se oponía al de molécula ya que mol (de etimología latina, moles) venía

\section{REFERENCIAS BIBLIOGRÁFICAS}

AINLEY, D., 1991, Mole catchers?, Education in Chemistry, 18-19.

BACHELARD, G., 1978. Lafilosofía del no. (Amorortu: Buenos Aires).

DE BERG, K.C., 1986a. Fundamental calculations with the mole, The Australian Science Teachers Journal, 32(1), pp. 29-36.

DE BERG, K.C., 1986b. Text book analysis of the mole and its underlying concepts, The Australian Science Teachers Journal, 31(1), pp. 33-43.

CAAMAÑO, A et al., 1983. Consideraciones sobre algunos errores conceptuales en el aprendizaje de la Química en el Bachillerato, Enseñanza de las Ciencias, 1, pp. 198-200.

DIERKS, W., 1981. Teaching the mole, European Journal of Science Education, 3 (2), pp. 145-158.

GRUPO ALKALI, 1990. Ideas de los alumnos acerca del mol. Estudio curricular, Enseñanza de las Ciencias, 8(2), pp. 111 118.

LLORENS, J.A.,1991. Comenzando a aprender química. (Visor: Madrid). a significar pila, montón o masa «grande» de una sustancia química que coincidia en número de gramos con la masa molecular relativa de la misma.

Así pues, la cantidad de sustancia química y el número de partículas son conceptos relacionados (como pueden estarlo la cantidad de sustancia química y la masa), pero ello no significa que sean idénticos. Es decir, la cantidad de sustancia química será una magnitud macroscópica (porción de sustancia), diferente a la masa y el volumen, que sirve para comparar cantidades de partículas pero que no las contabiliza directamente. En consecuencia, es distinta a la cantidad de entidades elementales.

En resumen, mientras se olvide Ia introducción de este concepto y no se abra un debate sobre su significado en la enseñanza de la química será bastante difícil idear estrategias de enseñanza que tengan por objeto conseguir un aprendizaje significativo del concepto de mol.

\section{Nota}

Este trabajo es una versión actualizada de la comunicación que con el mismo nombre se present6 en el III Congreso Internacional de Investigación en Didáctica de las Ciencias y de las Matemáticas (Santiago, 1989).
MIL.LS, I.M. et al., 1988. IUPAC. Quantities, units and symbols in physical chemistry. (Blackwell: Oxford).

NELSON,P.G.,1991. The elusive mole, Education in Chemistry, pp. 103-104.

OTERO, J.,1985. Assimilation problems in traditional representation of scientific knowledge, European Journal of Science Education, 7(4), pp. 361-369.

PIAGET, J.,1970. La epistemología genélica. (Ariel: Barcelona).

ROWELL, J.A. y DAWSON, C.J.,1989. Towards an integrated theory and practice for science teaching, Studies in Science Education, 16, pp. 47-73.

ROWELL, J.A. y DAWSON, C.J.,1980. Mountain or mole hill: can cognitive psychology reduce the dimensions of conceptual problems in classroom practice?, Science Education, pp. 693708.

SHAYER,M. y ADEY,Ph., 1984.LaCiencia de enseñar Ciencias. (Narcea: Madrid).

TOULMIN, \$., 1977. La comprension humana. I: el uso colectivo y la evolución de los conceptos. (Alianza: Madrid).

VUIK, R.,1981. Overview and critique of Piaget's genetic epistemology, 1965-1980. (Academic Press: Londres). 


\section{ANEXO 1}

\section{Prueba 1}

En los dibujos que aparecen a continuación se han representado pequeños trozos de sustancias con sus correspondientes átomos, para que compareis las cantidades de sustancias en los tres casos que se mencionan a continuación.

Para responder a esta prueba habrás de indicar dónde es mayor la cantidad de sustancia en cada caso (señala con una cruz la respuesta que consideras correcta) y explica las razones de tu eleccion.

\section{Primer caso}

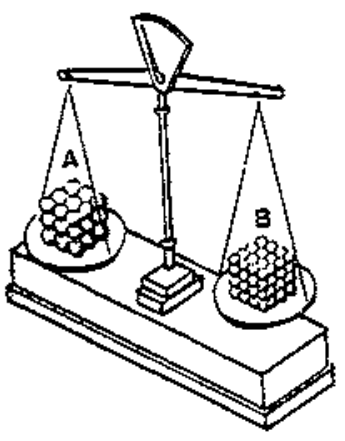

Es mayor la cantidad de sustancia en la A.

Es mayor la cantidad de sustancia en la B.

Es igual en las dos.

No lo sé.

\section{Explicación}

\section{Prueba 2}

Imagínate que sobre la mesa tenemos dos montones de cobre y azufre:
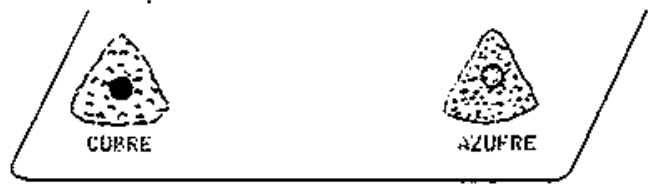

Sabiendo que cada átomo de cobre pesa el doble que cada átomo de azufre, ¿qué peso hay que coger de cada sustancia para que haya el mismo número de átomos de ambas?

Marca con una cruz la respuesta que consideras correcta:

El mismo peso de ambas.

Doble peso de cobre que de azufre.

Doble peso de azufre que de cobre.

Otra respuesta:

No lo sé.

\section{Explicación}

